\title{
Results of 10-year utilization of SPF (specific pathogen free) pigs at the Station of Pig Pathology of Ploufragan (France)
}

\author{
R. CARIOLET \\ Ministère de l'Agriculture, Station de Pathologie Porcine \\ B.P. 9, Les Croix, 22440 Ploufragan
}

Specific pathogen free piglets are used in the Station of Pig Pathology at Ploufragan (France) to study experimental infections. These animals are obtained either by aseptic hysterectomy and are then kept in isolators, or by natural delivery followed by rearing in a pig herd protected from undesirable contaminations. The quality of these piglets, their utilization for experimental studies, their growth and breeding performance over a 10-year period are reported and discussed as well as the problems related to the maintenance of their health status.

\section{Inventory of the bacterial flora in the pig respiratory system. Choice of the sample collection method for a better diagnosis and control of respiratory diseases}

\author{
F. MADEC *, Marylène KOBISCH *, Marguerite LE MENEC ** \\ * Ministère de l'Agriculture, Station de Pathologie Porcine \\ B.P. 9, Les Croix, 22440 Ploufragan \\ ** Laboratoire Départemental d'Analyses, B.P. 14, 22021 Saint-Brieuc Cedex
}

\begin{abstract}
A study was made in 20 breeding-finishing pig herds to determine the origin of sudden losses attributed to a septicemia induced by Streptococcus suis type 2. Bacteriological studies were made on several samples collected with different methods. The purpose of this study was to compare the adequacy of the collection methods to detect Streptococcus suis and the main pneumotrope agents in the herds. Samples were collected either from the heads and lungs of bacon pigs at the slaughter house or from 9-10 week old piglets selected at random in each herd and autopsied at the laboratory, or from nasal swabs made in sows and in piglets. Pasteurella multocida was mainly isolated in the damaged lungs of bacon pigs as well as Mycoplasma hyopneumoniae. On the other hand, the heads and particularly the tonsils of bacon pigs at slaughter turned out to be the best collection site for Streptococcus suis. Samples collected in the respiratory organs of sacrificed piglets did not give an accurate account of the health condition of the herd concerning Pasteurella multocida, Haemophilus pleuropneumoniae, Streptococcus suis and Mycoplasma hyopneumoniae. On the other hand, Bordetella bronchiseptica and especially Haemophilus parasuis were frequently isolated in those piglets. In sows, nasal swabs gave positive results for the different bacteria, but the frequencies of isolation were low. In 9-10 week old piglets, nasal swabs led to the same results as in the 3 autopsied piglets and thus could be an alternative when autopsy is not possible. It was concluded that to have an accurate estimation of the pneumotrope agents in a breeding-finishing herd it is advisable to get samples from at least 5 heads and 5 damaged lungs at the slaughter house and to complete with nasal swabs from 5 piglets.
\end{abstract}

